\title{
Application of The Extended Promethee II (EXPROM II) For International Student Exchange Selection
}

\author{
M Mesran ${ }^{1}$, Efori Buulolo ${ }^{1}$, Garuda Ginting ${ }^{2}$, Abdul Karim ${ }^{2}$ \\ \{m.mesra@gmail.com\} \\ ${ }^{1}$ Department Of Computer Science, STMIK Budi Darma, North Sumatera, Indonesia \\ ${ }^{2}$ Department Of Computer Science, AMIK STIEKOM Sumatera Utara, North Sumatera, Indonesia
}

\begin{abstract}
Students are learners who are on the bench of formal education. The role of schools in motivating learners to have a broader perspective is certainly needed, in addition, it should also be supported by the government of the Republic of Indonesia. International student exchange in this case strongly motivates students to be more active in learning. But the main problem, not all students get the opportunity to follow. Therefore, a decision support system that can be used for selection of student learners who will participate in an international student exchange program is required. Extended Method Promethee II (EXPROM II) is a method that is able to compare between each learner in multi-criteria. The use of EXPROM II, capable of producing more effective and more accurate results.
\end{abstract}

Keywords: EXPROM II, Extended Promethee II, International Student Exchange

\section{Introduction}

Students are people who participate in a learning process conducted by a unit of primary education level. Basic education, in this case, namely primary school, junior high school, and high school. In one region, or country, the students are one of the most important assets and must be managed properly and professionally. Students are the next generation of a nation, which will be useful in the future development of a nation. Currently, the education pattern in Indonesia is well managed and professionally managed, following the uniformity in the unit of education level, and at the end of the learning process will take the test, which is known as National Examination. But to increase the knowledge of learners is not only done on education bench only. Many factors can increase the knowledge of learners[1], can be extracurricular activities held both at school and outside the school, comparative studies to schools outside the province or international schools, student exchange programs and so forth.

Student exchange is a program conducted by various countries and one form of direct learning given to learners in school. The purpose of the exchange of students, so that learners have knowledge of the international world so as to add insight from a learner. Besides, other benefits of this program, namely the understanding of different perspectives of different cultures and communities, self-development that leads to an increase in self-belief, and able to develop awareness of group dynamics.

For that purpose, the government establishes an international student exchange system for students from a province. For Indonesian students, especially North Sumatra, Medan, there 
are certain criteria that must be fulfilled in following the selection process held by Medan City Government. Selection of student exchanges conducted by the city of Medan, of course not easy because there are several stages or processes that are tailored to the criteria that have been established based on existing alternatives.

Implementation [2] of information systems-based decision support system[3]-[7] help the city of Medan in the process of producing more effective and accurate decisions. Many decision-makers use decision support systems to support the decisions they set, but not necessarily replace the decisions they have. Decision support systems are able to provide problem-solving results based on various predefined criteria [8]. In the application of this computer-based system using methods in performing analysis of decision-making such as Elimination and Choice Translation Reality (ELECTRE), Preference Rank Organization Organization for Enrichment of Evaluation (PROMETHEE), Vlse Kriterijumska Optimizacija Kompromisno Resenje (VIKOR), Analytical Hierarchy Process AHP) [9], [10].

The use of TOPSIS method can be used for decision making as done by Garuda Ginting [6]. In his research Rajesh Attri (2015) used the Preference Selection Index (PSI) method for the selection of production design[11]. Madic 2017 uses Preference Selection for the determination of laser cutting process conditions [12]. In a study conducted by Teuku Mufizar in 2017, using several criteria in the selection of student exchanges such as the TOEFL Score, Ranking, Interview Value, Indonesian knowledge, artistic value, Australian knowledge, personality value, from research results in the processor the TOEFL Score and Ranking criteria have significant weighting to the calculation result[13]. The use of EXPROM II method managed to give a decision about a product that deserves a discount [14].

\section{Theory}

\subsection{Student}

Students are a designation given to learners who follow formal education process at the primary, secondary and top level. Etymologically, the learner gets the teaching of a teacher, and the terminology of the learner will undergo change, development and require guidance and direction to form personality.

\subsection{International Student Exchange}

International student exchange is a program often done by the government of the Republic of Indonesia, with the aim of increasing knowledge for learners. There are three (3) benefits gained for students who follow this program, namely:

1. Have a soul of independence and increased maturity in students.

2. Will more appreciate the relationship between humans, as well.

3. Increased ability in foreign language

\subsection{The Extended Promethee II Method}

The Extended Promethee II (EXPROM II) is a development of the Promethee II method, developed by Diakoulaki and Koumoutsosa (Fadlina et al., 2017). Development is done on the comparison [15] of deviation or distance between alternatives in pairs for each criterion. In the 
EXPROM II method, there are two preference indexes obtained from the comparison between the paired alternate values. The first preference index is known as the Weak Preferences Index and the second is known as Strict Preference Index, this is where searching process [16], [17] were known and get the results.

The Total Preferences Index is calculated from the reduction of the Weak Preferences Index and Strict Preferences Index values, thus providing a more accurate measure of the preference of one alternative with another alternative on all criteria. The calculation of net flow in an alternative is determined based on the sum of leaving and entering flow. EXPROM II generates alternate rankings based on the net flow value of each alternative so as to provide the best alternative with the highest net flow.

The settlement step is using EXPROM II[18], as follows:

1. Creating a decision matrix

The decision matrix is a matrix consisting of an alternative value $i$ for each criterion $j$.

2. Normalization of Decision Matrix.

For the Benefit Criteria:

$\mathrm{r}_{\mathrm{ij}}=\frac{\left[\mathrm{x}_{\mathrm{ij}}-\min \left(\mathrm{x}_{\mathrm{ij}}\right)\right]}{\left[\max \left(\mathrm{x}_{\mathrm{ij}}\right)-\min \left(\mathrm{x}_{\mathrm{ij}}\right)\right]}$

For the Cost Criteria:

$\mathrm{r}_{\mathrm{ij}}=\frac{\left[\max \left(\mathrm{x}_{\mathrm{ij}}\right)-\mathrm{x}_{\mathrm{ij}}\right]}{\left[\max \left(\mathrm{x}_{\mathrm{ij}}\right)-\min \left(\mathrm{x}_{\mathrm{ij}}\right)\right]}$

Where $\quad$ rij $=$ normalized matrix

$i=$ alternative

$j=$ criteria

3. Calculates evaluative differences from alternative $i^{\text {th }}$ with other alternatives. This step involves calculating the difference in criterion value $\left(\mathrm{d}_{\mathrm{j}}\right)$ between different alternatives pair-wise.

4. Determination of Preferences Function $\operatorname{Pj}\left(i, i^{\prime}\right)$.

There are six main types of preference functions, for example, ordinary criteria, $U$ shape formulas, form $\mathrm{V}$ criteria, level criteria, V-shrink criteria and Gaussian criteria. The simplest form of the preferences function (ordinary criteria) is applied here, the $\mathrm{Pj}$ ( $\mathrm{i}, \mathrm{i}$ ') Preferences Function Equation is given below:

$P_{i j}\left(i, i^{\prime}\right)=0$ if $r_{i j} \leq r_{i j}$

$P_{i j}\left(i, i^{\prime}\right)=\left(r_{i j}-r_{i^{\prime} j}\right)$ if $r_{i j}>r_{i^{\prime} j}$

where $\mathrm{P}_{\mathrm{ij}}=$ Preferences Function

$I=$ alternative

i' = alternatives to be paired with

5. Calculating Weak Preference Index (WP)

Calculating the weak preference index should consider the criteria with different weight values for each criterion using the following equation:

$\mathrm{WP}\left(\mathrm{i}, \mathrm{i}^{\prime}\right)=\left[\sum_{\mathrm{j}=1}^{\mathrm{n}} \mathrm{w}_{\mathrm{j}} \times \mathrm{P}_{\mathrm{j}}(\mathrm{i}, \mathrm{i})\right] / \sum_{\mathrm{j}=1}^{\mathrm{n}} \mathrm{w}_{\mathrm{j}}$

where WP $=$ Weak Preference Index $\mathrm{n}=$ number of criteria 
$\mathrm{w}_{\mathrm{j}}=$ weighting $\mathrm{j}$ criteria

6. Calculating Strict Preferences Index (SP j (i,i') ).

The determination of a strong preference function or SP j (i, i') is based on the ratio of the difference values $\left(\mathrm{dm}_{\mathrm{j}}\right)$ to the range of values as defined by the evaluation of the entire alternative set for the criteria.

$\mathrm{SP}_{\mathrm{j}}\left(\mathrm{i}, \mathrm{i}^{\prime}\right)=\left[\max \left(0, \mathrm{~d}_{\mathrm{j}}-\mathrm{L}_{\mathrm{j}}\right)\right] /\left[\mathrm{dm}_{\mathrm{j}}-\mathrm{L}_{\mathrm{j}}\right]$

where $: \mathrm{SPj}=$ Strict Preference index $\mathrm{j}$

$\mathrm{dj}=$ rij-ri'j

$\mathrm{dmj}=1$

$\mathrm{Lj}=0$

7. Computer the Strict Preference Index (SP)

In calculating the strong preference index value (SP), use the following equation:

$\operatorname{SP}\left(i, i^{\prime}\right)=\left[\sum_{j=1}^{n} w_{j} \times \operatorname{SP}_{j}\left(i, i^{\prime}\right)\right] / \sum_{j=1}^{n} w_{j}$

where $\mathrm{SP}=$ Strict Preference Index

$\mathrm{n}=$ number of criteria

$\mathrm{w}_{\mathrm{j}}=$ weighting $\mathrm{j}$ criteria

8. Compute the value of Total Preferences TP(i, i')

For the calculation of Total Preference index value:

$\mathrm{TP}\left(\mathrm{i}, \mathrm{i}^{\prime}\right)=\operatorname{Min}\left[1, \mathrm{WP}\left(\mathrm{i}, \mathrm{i}^{\prime}\right)+\mathrm{SP}\left(\mathrm{i}, \mathrm{i}^{\prime}\right)\right]$

9. Determining Leaving and Entering Flow

Leaving Flow (Prositive):

$\varphi^{+}(i)=\frac{1}{m-1} \sum_{i^{\prime}=1}^{m} \operatorname{TP}\left(i, i^{\prime}\right)\left(i \neq i^{\prime}\right)$

Entering Flow (Negative)

$\varphi^{-}(\mathrm{i})=\frac{1}{\mathrm{~m}-1} \sum_{\mathrm{i}^{\prime}=1}^{\mathrm{m}} \mathrm{TP}\left(\mathrm{i}^{\prime}, \mathrm{i}\right)\left(\mathrm{i} \neq \mathrm{i}^{\prime}\right) \ldots \ldots \ldots \ldots$

where $\mathrm{i}=$ alternative

i' = paired alternatives

$\mathrm{m}=$ number of alternative

ln $=$ number of criteria

Leaving Flow reveals how many alternatives dominate other alternatives, while the Entering Flow shows how many alternatives are dominated by other alternatives.

10. Calculate the Net Flow

Net flow calculation $\varphi(i)$ for each alternative:

$\varphi(i)=\varphi^{+}(\mathrm{i})-\varphi^{-}(\mathrm{i})$

11. Determine the rangking.

The ranking of all alternatives considered depends on the values of $\varphi$ (i). The higher the value of $\varphi(i)$, the better the alternative.

After the above step produces a decision, then the best alternative is the alternative that has the highest of net flow $(\varphi)$. 


\section{Result \& Discussion}

Processing on decision support systems by applying the EXPROM II method requires criteria that are used as a reference to compare alternatives. The criteria used in comparison to alternatives include TOEFL score, ranking, Traditional Dance, Interview, Personality. Of the five (5) criteria are all with the type of beneficial criteria which means the greatest value, then the alternative becomes better to be compared with others.

From the explanation can be seen the required criteria as follows:

Table 1: Criteria

\begin{tabular}{lcc}
\hline \multicolumn{1}{c}{ Criteria } & Weight & Type \\
\hline TOEFL Score $\left(\mathrm{C}_{1}\right)$ & $25 \%$ & Benefit \\
Rank $\left(\mathrm{C}_{2}\right)$ & $20 \%$ & Benefit \\
Traditional Dance Art $\left(\mathrm{C}_{3}\right)$ & $30 \%$ & Benefit \\
Interview $\left(\mathrm{C}_{4}\right)$ & $10 \%$ & Benefit \\
Personality $\left(\mathrm{C}_{5}\right)$ & $15 \%$ & Benefit \\
\hline
\end{tabular}

The alternative consists of five (5) students drawn from several different schools, namely Taufik Ismail $\left(A_{1}\right)$, Suryanto $\left(A_{2}\right)$, Chairunnisah Putri $\left(A_{3}\right)$, Muhammad Rifqy $\left(A_{4}\right)$, Damar Wulan $\left(\mathrm{A}_{5}\right)$. In table 2 below is a match rating between alternatives and criteria.

Table 2: Alternative match Rating and Criteria

\begin{tabular}{cccccc}
\hline Alternatif & $\mathrm{C}_{1}$ & $\mathrm{C}_{2}$ & $\mathrm{C}_{3}$ & $\mathrm{C}_{4}$ & $\mathrm{C}_{5}$ \\
\hline $\mathrm{A}_{1}$ & 450 & 2 & 90 & 80 & 90 \\
$\mathrm{~A}_{2}$ & 500 & 2 & 86 & 80 & 90 \\
$\mathrm{~A}_{3}$ & 490 & 1 & 90 & 90 & 80 \\
$\mathrm{~A}_{4}$ & 530 & 2 & 90 & 80 & 85 \\
$\mathrm{~A}_{5}$ & 550 & 3 & 85 & 85 & 90 \\
\hline
\end{tabular}

The first stage after defining alternatives and criteria, forms the decision matrix $\mathrm{x}_{\mathrm{ij}}$, as follows.

$X i j=\left[\begin{array}{lllll}450 & 2 & 90 & 80 & 90 \\ 500 & 2 & 86 & 80 & 90 \\ 490 & 1 & 90 & 90 & 80 \\ 530 & 2 & 90 & 80 & 85 \\ 550 & 3 & 85 & 85 & 90\end{array}\right]$

From the matrix $\mathrm{x}_{\mathrm{ij}}$, then normalize into matrix $\mathrm{r}_{\mathrm{ij}}$.

$$
\begin{array}{lll}
\mathrm{C}_{1}=\text { TOEFL Score } & \mathrm{C}_{2}=\text { Rank } & \mathrm{C}_{3}=\text { Traditional Dance Art } \\
R 1,1=\frac{[450-450]}{[550-450]}=0 & R 1,2=\frac{[2-1]}{[3-1]}=0,5 & R 1,3=\frac{[90-85]}{[90-85]}=1 \\
R 2,1=\frac{[500-450]}{[550-450]}=0,5 & R 2,2=\frac{[2-1]}{[3-1]}=0,5 & R 2,3=\frac{[86-85]}{[90-85]}=0,2 \\
R 3,1=\frac{[490-450]}{[550-450]}=0,4 & R 3,2=\frac{[1-1]}{[3-1]}=0 & R 3,3=\frac{[90-85]}{[90-85]}=1
\end{array}
$$




$$
\begin{aligned}
& R 4,1=\frac{[530-450]}{[550-450]}=0,8 \quad R 4,2=\frac{[2-1]}{[3-1]}=0,5 \quad R 4,3=\frac{[90-85]}{[90-85]}=1 \\
& R 5,1=\frac{[550-450]}{[550-450]}=1 \quad R 5,2=\frac{[3-1]}{[3-1]}=1 \quad R 5,3=\frac{[85-85]}{[90-85]}=0 \\
& \mathrm{C}_{4}=\text { Interview } \quad \mathrm{C}_{5}=\text { Personality } \\
& R 1,4=\frac{[80-80]}{[90-80]}=0 \quad R 1,5=\frac{[90-80]}{[90-80]}=1 \\
& R 2,4=\frac{[80-80]}{[90-80]}=0 \quad R 2,5=\frac{[90-80]}{[90-80]}=1 \\
& R 3,4=\frac{[90-80]}{[90-80]}=1 \quad R 3,5=\frac{[80-80]}{[90-80]}=0 \\
& R 4,4=\frac{[80-80]}{[90-80]}=0 \quad R 4,5=\frac{[85-80]}{[90-80]}=0,5 \\
& R 5,4=\frac{[85-80]}{[90-80]}=0,5 \quad R 5,5=\frac{[90-80]}{[90-80]}=1
\end{aligned}
$$

The processing of matrix $\mathrm{x}_{\mathrm{ij}}$, resulting in a normalized matrix $\mathrm{r}_{\mathrm{ij}}$, as follows.

$$
R i j=\left[\begin{array}{lllll}
0 & 0,5 & 1 & 0 & 1 \\
0,5 & 0,5 & 0,2 & 0 & 1 \\
0,4 & 0 & 1 & 1 & 0 \\
0,8 & 0,5 & 1 & 0 & 0,5 \\
1 & 1 & 0 & 0,5 & 1
\end{array}\right]
$$

Next calculate the value of Preferences $P_{j}\left(i, i^{\prime}\right)$ using equations 3 and 4 .

For $\mathrm{C}_{1}$, pairs-wise matrix:

if $0 \leq 0,5$ True, then $P_{1}(1,2)=0$

if $0 \leq 0,4$ True, then $P_{1}(1,3)=0$

if $0 \leq 0,8$ True, then $P_{1}(1,4)=0$

if $0 \leq 1$ True, then $P_{1}(1,5)=0$

if $0,5 \leq 0$ False Else if $0,5>0$ True, then $P_{1}(2,1)=0,5-0=0,5$

if $0,5 \leq 0,4$ False Else if $0,5>0,4$ True, then $P_{1}(2,3)=0,5-4=0,1$

if $0,5 \leq 0,8$ True, then $P_{1}(2,4)=0$

if $0,5 \leq 1$ True, then $P_{1}(2,5)=0$

if $0,4 \leq 0$ False Else if $0,4>0$ True, then $P_{1}(3,1)=0,4-0=0,4$

if $0,4 \leq 0,5$ True, then $P_{1}(3,2)=0$

if $0,4 \leq 0,4$ True, then $P_{1}(3,4)=0$

if $0,4 \leq 1$ True, then $P_{1}(3,5)=0$

if $0,8 \leq 0$ False Else if $0,8>0$ True, then $P_{1}(4,1)=0,8-0=0,8$

if $0,8 \leq 0,5$ False Else if $0,8>0,5$ True, then $P_{1}(4,2)=0,8-0,5=0,3$

if $0,8 \leq 0,4$ False Else if $0,8>0,4$ True, then $P_{1}(4,3)=0,8-0,4=0,4$

if $0,8 \leq 1$ True, then $P_{1}(4,5)=0$

if $1 \leq 0$ False Else if $1>0$ True, then $P_{1}(5,1)=1-0=1$

if $1 \leq 0,5$ False Else if $1>0,5$ True, then $P_{1}(5,2)=1-0,5=0,5$ 
if $1 \leq 0,4$ False Else if $1>0,4$ True, then $P_{1}(5,3)=1-0,4=0,6$ if $1 \leq 0,8$ False Else if $1>0,8$ True, then $P_{1}(5,4)=1-0,8=0,2$ For $\mathrm{C}_{2}$, pairs-wise matrix:

if $0,5 \leq 0,5$ True, then $P_{2}(1,2)=0$

if $0,5 \leq 0$ False, Else if $0,5>0$ True, then $P_{2}(1,3)=0,5-0=0,5$ if $0,5 \leq 0,5$ True, then $P_{2}(1,4)=0$

if $0,5 \leq 1$ True, then $P_{2}(1,5)=0$

if $0,5 \leq 0,5$ True, then $P_{2}(2,1)=0$

if $0,5 \leq 0$ False, Else if $0,5>0$ True, then $P_{2}(2,3)=0,5-0=0,5$

if $0,5 \leq 0,5$ True, then $P_{2}(2,4)=0$

if $0,5 \leq 1$ True, then $P_{2}(2,5)=0$

if $0 \leq 0,5$ True, then $P_{2}(3,1)=0$

if $0 \leq 0,5$ True, then $P_{2}(3,2)=0$

if $0 \leq 0,5$ True, then $P_{2}(3,4)=0$

if $0 \leq 1$ True, then $P_{2}(3,5)=0$

if $0,5 \leq 0,5$ True, then $P_{2}(4,1)=0$

if $0,5 \leq 0,5$ True, then $P_{2}(4,2)=0$

if $0,5 \leq 0$ False, Else if $0,5>0$ True, then $P_{2}(4,3)=0,5-0=0,5$

if $0,5 \leq 1$ True, then $P_{2}(4,5)=0$

if $1 \leq 0,5$ False Else if $1>0,5$ True, then $P_{2}(5,1)=1-0,5=0,5$

if $1 \leq 0,5$ False Else if $1>0,5$ True, then $P_{2}(5,2)=1-0,5=0,5$

if $1 \leq 0$ False Else if $1>0$ True, then $P_{2}(5,3)=1-0=1$

if $1 \leq 0,5$ False Else if $1>0,5$ True,

then $P_{2}(5,4)=1-0,5=0,5$

For $\mathrm{C}_{3}$, pairs-wise matrix:

if $1 \leq 0,2$ False, Else if $1>0,2$ True,

then $P_{3}(1,2)=1-0,2=0,8$

if $1 \leq 1$ True, then $P_{3}(1,3)=0,5-0=0,5$

if $1 \leq 1$ True, then $P_{3}(1,4)=0$

if $1 \leq 0$ False, Else if $1>0$ True,

then $P_{3}(1,5)=1-0=1$

if $0,2 \leq 1$ True, then $P_{3}(2,1)=0$

if $0,2 \leq 1$ True, then $P_{3}(2,3)=0$

if $0,2 \leq 1$ True, then $P_{3}(2,4)=0$

if $0,2 \leq 0$ False, Else if $0,2>0$ True,

then $P_{3}(2,5)=0,2-0=0,2$

if $1 \leq 1$ True, then $P_{3}(3,1)=0$

if $1 \leq 0,2$ False, Else if $1>0,2$ True,

then $P_{3}(3,2)=1-0,2=0,8$

if $1 \leq 1$ True, then $P_{3}(3,4)=0$

if $1 \leq 0$ False, Else if $1>0$ True,

then $P_{3}(3,5)=1-0=1$

if $1 \leq 1$ True, then $P_{3}(4,1)=0$

if $1 \leq 0,2$ False, Else if $1>0,2$ True, then $P_{3}(4,2)=1-0,2=0,8$

if $1 \leq 1$ True, then $P_{3}(4,3)=0$

if $1 \leq 0$ False, Else if $1>0$ True, 


$$
\begin{aligned}
& \text { then } P_{3}(4,5)=1-0=1 \\
& \text { if } 0 \leq 1 \text { True, then } P_{3}(5,1)=0 \\
& \text { if } 0 \leq 0,2 \text { True, then } P_{3}(5,2)=0 \\
& \text { if } 0 \leq 1 \text { True, then } P_{3}(5,3)=0 \\
& \text { if } 0 \leq 1 \text { True, then } P_{3}(5,4)=0
\end{aligned}
$$

For $\mathrm{C}_{4}$, pairs-wise matrix:

$$
\begin{aligned}
& \text { if } 0 \leq 0 \text { True, then } P_{4}(1,2)=0 \\
& \text { if } 0 \leq 1 \text { True, then } P_{4}(1,3)=0 \\
& \text { if } 0 \leq 0 \text { True, then } P_{4}(1,4)=0 \\
& \text { if } 0 \leq 0,5 \text { True, then } P_{4}(1,5)=0 \\
& \text { if } 0 \leq 0 \text { True, then } P_{4}(2,1)=0 \\
& \text { if } 0 \leq 1 \text { True, then } P_{4}(2,3)=0 \\
& \text { if } 0 \leq 0 \text { True, then } P_{4}(2,4)=0 \\
& \text { if } 0 \leq 0,5 \text { True, then } P_{4}(2,5)=0 \\
& \text { if } 1 \leq 0 \text { False, Else if } 1>0 \text { True, then } P_{4}(3,1)=1-0=1 \\
& \text { if } 1 \leq 0 \text { False, Else if } 1>0 \text { True, } \\
& \text { then } P_{4}(3,2)=1-0=1 \\
& \text { if } 1 \leq 0 \text { False, Else if } 1>0 \text { True, } \\
& \text { then } P_{4}(3,4)=1-0=1 \\
& \text { if } 1 \leq 0,5 \text { False, Else if } 1>0,5 \text { True, } \\
& \text { then } P_{4}(3,5)=1-0,5=0,5 \\
& \text { if } 0 \leq 0 \text { True, then } P_{4}(4,1)=0 \\
& \text { if } 0 \leq 0 \text { True, then } P_{4}(4,2)=0 \\
& \text { if } 0 \leq 1 \text { True, then } P_{4}(4,3)=0 \\
& \text { if } 0 \leq 0,5 \text { True, then } P_{4}(4,5)=0 \\
& \text { if } 0,5 \leq 0 \text { False, Else if } 0,5>0 \text { True, then } P_{4}(5,1)=0,5-0=0,5 \\
& \text { if } 0,5 \leq 0 \text { False, Else if } 0,5>0 \text { True, then } P_{4}(5,2)=0,5-0=0,5 \\
& \text { if } 0,5 \leq 1 \text { True, then } P_{4}(5,3)=0 \\
& \text { if } 0,5 \leq 0 \text { False, Else if } 0,5>0 \text { True, } \\
& \text { then } P_{4}(5,4)=0,5-0=0,5
\end{aligned}
$$

For $\mathrm{C}_{5}$, pairs-wise matrix:

if $1 \leq 1$ True, then $P_{5}(1,2)=0$

if $1 \leq 0$ False, Else if $1>0$ True, then $P_{5}(1,3)=1-0=1$

if $1 \leq 0,5$ False, Else if $1>0,5$ True,

then $P_{5}(1,4)=1-0,5=0,5$

if $1 \leq 1$ True, then $P_{5}(1,5)=0$

if $1 \leq 1$ True, then $P_{5}(2,1)=0$

if $1 \leq 0$ False, Else if $1>0$ True, then $P_{5}(2,3)=1-0=1$

if $1 \leq 0,5$ False, Else if $1>0,5$ True,

then $P_{5}(2,4)=1-0,5=0,5$

if $1 \leq 1$ True, then $P_{5}(2,5)=0$

if $0 \leq 1$ True, then $P_{5}(3,1)=0$

if $0 \leq 1$ True, then $P_{5}(3,2)=0$

if $0 \leq 0,5$ True, then $P_{5}(3,4)=0$

if $0 \leq 1$ True, then $P_{5}(3,5)=0$ 
if $0,5 \leq 1$ True, then $P_{5}(4,1)=0$

if $0,5 \leq 1$ True, then $P_{5}(4,2)=0$

if $0,5 \leq 0$ False, Else if $0,5>0$ True, then $P_{5}(4,3)=0,5-0=0,5$

if $0,5 \leq 1$ True, then $P_{5}(4,5)=0$

if $1 \leq 1$ True, then $P_{3}(5,1)=0$

if $1 \leq 1$ True, then $P_{3}(5,2)=0$

if $1 \leq 0$ False, Else if $1>0$ True, then $P_{3}(5,3)=1-0=1$

if $1 \leq 0,5$ False, Else if $1>0,5$ True,

then $P_{3}(5,4)=1-0,5=0,5$

Recapitulation of results from the comparison of each alternative in each criterion can be seen in table 3.

Table 3. Comparison results for each alternative in each criteria column

\begin{tabular}{cccccc}
\hline Alternative & $\mathrm{C}_{1}$ & $\mathrm{C}_{2}$ & $\mathrm{C}_{3}$ & $\mathrm{C}_{4}$ & $\mathrm{C}_{5}$ \\
\hline $\mathrm{P}_{1}(1,2)$ & 0 & 0 & 0,8 & 0 & 0 \\
$\mathrm{P}_{1}(1,3)$ & 0 & 0,5 & 0 & 0 & 1 \\
$\mathrm{P}_{1}(1,4)$ & 0 & 0 & 0 & 0 & 0,5 \\
$\mathrm{P}_{1}(1,5)$ & 0 & 0 & 1 & 0 & 0 \\
$\mathrm{P}_{2}(2,1)$ & 0,5 & 0 & 0 & 0 & 0 \\
$\mathrm{P}_{2}(2,3)$ & 0,1 & 0,5 & 0 & 0 & 1 \\
$\mathrm{P}_{2}(2,4)$ & 0 & 0 & 0 & 0 & 0,5 \\
$\mathrm{P}_{2}(2,5)$ & 0 & 0 & 0,2 & 0 & 0 \\
$\mathrm{P}_{3}(3,1)$ & 0,4 & 0 & 0 & 1 & 0 \\
$\mathrm{P}_{3}(3,2)$ & 0 & 0 & 0,8 & 1 & 0 \\
$\mathrm{P}_{3}(3,4)$ & 0 & 0 & 0 & 1 & 0 \\
$\mathrm{P}_{3}(3,5)$ & 0 & 0 & 1 & 0,5 & 0 \\
$\mathrm{P}_{4}(4,1)$ & 0,8 & 0 & 0 & 0 & 0 \\
$\mathrm{P}_{4}(4,2)$ & 0,3 & 0 & 0,8 & 0 & 0 \\
$\mathrm{P}_{4}(4,3)$ & 0,4 & 0,5 & 0 & 0 & 0,5 \\
$\mathrm{P}_{4}(4,5)$ & 0 & 0 & 1 & 0 & 0 \\
$\mathrm{P}_{5}(5,1)$ & 1 & 0,5 & 0 & 0,5 & 0 \\
$\mathrm{P}_{5}(5,2)$ & 0,5 & 0,5 & 0 & 0,5 & 0 \\
$\mathrm{P}_{5}(5,3)$ & 0,6 & 1 & 0 & 0 & 1 \\
$\mathrm{P}_{5}(5,4)$ & 0,2 & 0,5 & 0 & 0,5 & 0,5 \\
\hline
\end{tabular}

The next step calculates the Value Index of the Weak Preferences (WP) using equation 5.

$\mathrm{WP}_{1,2}=((0,25 * 0)+(0,2 * 0)+(0,3 * 0,8)+(0,1 * 0)+$

$$
(0,15 * 0)) / 1
$$

$$
=0,24
$$

$\mathrm{WP}_{1,3}=((0,25 * 0)+(0,2 * 0,5)+(0,3 * 0)+(0,1 * 0)+$ $(0,15 * 1)) / 1$

$=0,25$

$\mathrm{WP}_{1,4}=((0,25 * 0)+(0,2 * 0)+(0,3 * 0)+(0,1 * 0)+$

$(0,15 * 0,5)) / 1$

$=0,075$

$\mathrm{WP}_{1,5}=((0,25 * 0)+(0,2 * 0)+(0,3 * 1)+(0,1 * 0)+$

$(0,15 * 0)) / 1$

$=0,3$

$\mathrm{WP}_{2,1}=((0,25 * 0,5)+(0,2 * 0)+(0,3 * 0)+(0,1 * 0)+$ $(0,15 * 0)) / 1$ 


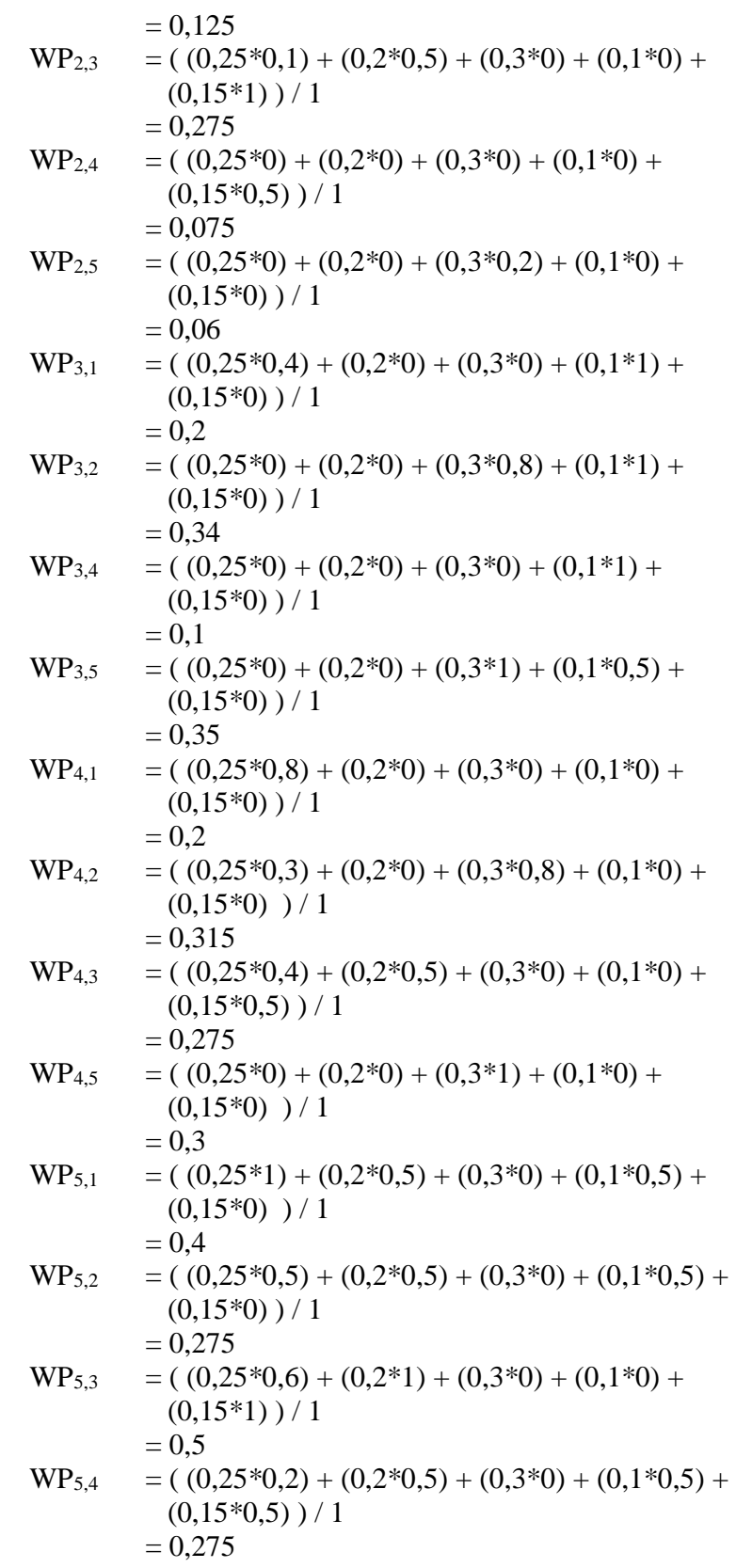

Here is the result table of the weak preference value index (WP).

Table 4. The Weak Preference (WP)

\begin{tabular}{cc}
\hline Alternative & $\mathrm{WP}\left(\mathrm{i}, \mathrm{i}^{\prime}\right)$ \\
\hline $\mathrm{WP}_{1,2}$ & 0,24 \\
$\mathrm{WP}_{1,3}$ & 0,25 \\
\hline
\end{tabular}




\begin{tabular}{cc}
\hline Alternative & $\mathrm{WP}(\mathrm{i}, \mathrm{i})$ \\
\hline $\mathrm{WP}_{1,4}$ & 0,075 \\
$\mathrm{WP}_{1,5}$ & 0,3 \\
$\mathrm{WP}_{2,1}$ & 0,125 \\
$\mathrm{WP}_{2,3}$ & 0,275 \\
$\mathrm{WP}_{2,4}$ & 0,075 \\
$\mathrm{WP}_{2,5}$ & 0,06 \\
$\mathrm{WP}_{3,1}$ & 0,2 \\
$\mathrm{WP}_{3,2}$ & 0,34 \\
$\mathrm{WP}_{3,4}$ & 0,1 \\
$\mathrm{WP}_{3,5}$ & 0,35 \\
$\mathrm{WP}_{4,1}$ & 0,2 \\
$\mathrm{WP}_{4,2}$ & 0,315 \\
$\mathrm{WP}_{4,3}$ & 0,275 \\
$\mathrm{WP}_{4,5}$ & 0,3 \\
$\mathrm{WP}_{5,1}$ & 0,4 \\
$\mathrm{WP}_{5,2}$ & 0,275 \\
$\mathrm{WP}_{5,3}$ & 0,5 \\
$\mathrm{WP}_{5,4}$ & 0,275 \\
\hline
\end{tabular}

Next, use the 7th equation, to calculated strict preference index value, as result in table 5 .

Table 5. The Strict Preference (SP)

\begin{tabular}{cc}
\hline Alternative & $\mathrm{SP}\left(\mathrm{i}, \mathrm{i}^{\prime}\right)$ \\
\hline $\mathrm{SP}_{1,2}$ & 0,24 \\
$\mathrm{SP}_{1,3}$ & 0,25 \\
$\mathrm{SP}_{1,4}$ & 0,075 \\
$\mathrm{SP}_{1,5}$ & 0,3 \\
$\mathrm{SP}_{2,1}$ & 0,125 \\
$\mathrm{SP}_{2,3}$ & 0,275 \\
$\mathrm{SP}_{2,4}$ & 0,075 \\
$\mathrm{SP}_{2,5}$ & 0,06 \\
$\mathrm{SP}_{3,1}$ & 0,2 \\
$\mathrm{SP}_{3,2}$ & 0,34 \\
$\mathrm{SP}_{3,4}$ & 0,1 \\
$\mathrm{SP}_{3,5}$ & 0,35 \\
$\mathrm{SP}_{4,1}$ & 0,2 \\
$\mathrm{SP}_{4,2}$ & 0,315 \\
$\mathrm{SP}_{4,3}$ & 0,275 \\
$\mathrm{SP}_{4,5}$ & 0,3 \\
$\mathrm{SP}_{5,1}$ & 0,4 \\
$\mathrm{SP}_{5,2}$ & 0,275 \\
$\mathrm{SP}_{5,3}$ & 0,5 \\
$\mathrm{SP}_{5,4}$ & 0,275 \\
\hline
\end{tabular}

From WP and SP values, then calculated the index value of total preference using the equation to 8 .

Table 6. The Total Preference Value (TP)

\begin{tabular}{cl}
\hline Alternative & \multicolumn{1}{c}{ Value } \\
\hline $\operatorname{TP}_{1,2}$ & Min $[1 ; 0,24+0,24]=0,48$ \\
$\operatorname{TP}_{1,3}$ & $\operatorname{Min}[1 ; 0,25+0,25]=0,5$ \\
$\operatorname{TP}_{1,4}$ & Min $[1 ; 0,075+0,075]=0,15$ \\
\hline
\end{tabular}




\begin{tabular}{cl}
\hline Alternative & \multicolumn{1}{c}{ Value } \\
\hline $\mathrm{TP}_{1,5}$ & Min $[1 ; 0,3+0,3]=0,6$ \\
$\mathrm{TP}_{2,1}$ & $\operatorname{Min}[1 ; 0,125+0,125]=0,25$ \\
$\mathrm{TP}_{2,3}$ & $\operatorname{Min}[1 ; 0,275+0,275]=0,55$ \\
$\mathrm{TP}_{2,4}$ & $\operatorname{Min}[1 ; 0,075+0,075]=0,15$ \\
$\mathrm{TP}_{2,5}$ & $\operatorname{Min}[1 ; 0,06+0,06]=0,12$ \\
$\mathrm{TP}_{3,1}$ & Min $[1 ; 0,2+0,2]=0,4$ \\
$\mathrm{TP}_{3,2}$ & $\operatorname{Min}[1 ; 0,34+0,34]=0,68$ \\
$\mathrm{TP}_{3,4}$ & Min $[1 ; 0,1+0,1]=0,2$ \\
$\mathrm{TP}_{3,5}$ & $\operatorname{Min}[1 ; 0,35+0,35]=0,7$ \\
$\mathrm{TP}_{4,1}$ & $\operatorname{Min}[1 ; 0,2+0,2]=0,4$ \\
$\mathrm{TP}_{4,2}$ & $\operatorname{Min}[1 ; 0,315+0,315]=0,63$ \\
$\mathrm{TP}_{4,3}$ & $\operatorname{Min}[1 ; 0,275+0,275]=0,55$ \\
$\mathrm{TP}_{4,5}$ & $\operatorname{Min}[1 ; 0,3+0,3]=0,6$ \\
$\mathrm{TP}_{5,1}$ & $\operatorname{Min}[1 ; 0,4+0,4]=0,8$ \\
$\mathrm{TP}_{5,2}$ & $\operatorname{Min}[1 ; 0,275+0,275]=0,55$ \\
$\mathrm{TP}_{5,3}$ & $\operatorname{Min}[1 ; 0,5+0,5]=1$ \\
$\mathrm{TP}_{5,4}$ & $\operatorname{Min}[1 ; 0,275+0,275]=0,55$ \\
\hline
\end{tabular}

From table 6 above, the following dominant aggregate matrix will be formed:

$\left[\begin{array}{lllll}- & 0,48 & 0,5 & 0,15 & 0,6 \\ 0,25 & - & 0,55 & 0,15 & 0,12 \\ 0,4 & 0,68 & - & 0,2 & 0,7 \\ 0,4 & 0,63 & 0,55 & - & 0,6 \\ 0,8 & 0,55 & 1 & 0,55 & -\end{array}\right]$

The next process is to find Leaving Flow and Entering Flow using equations 9 and 10, like the following:

Calculates the Leaving Flow:

$$
\begin{aligned}
\varphi 1^{+} & =\frac{1}{5-1}[0,48+0,5+0.15+0,6]=0,4235 \\
\varphi 2^{+} & =\frac{1}{5-1}[0,25+0,55+0,15+0,12]=0,2675 \\
\varphi 3^{+} & =\frac{1}{5-1}[0,4+0,68+0,2+0,7]=0,495 \\
\varphi 4^{+} & =\frac{1}{5-1}[0,4+0,63+0,55+0,6]=0,545 \\
\varphi 5^{+} & =\frac{1}{5-1}[0,8+0,55+1+0,55]=0,725
\end{aligned}
$$

Calculates the Entering Flow:

$$
\begin{aligned}
& \varphi 1^{-}=\frac{1}{5-1}[0,25+0,4+0,4+0,8]=0,4625 \\
& \varphi 2^{-}=\frac{1}{5-1}[0,48+0,68+0,63+0,55]=0,585 \\
& \varphi 3^{-}=\frac{1}{5-1}[0,5+0,55+0,55+1]=0,65 \\
& \varphi 4^{-}=\frac{1}{5-1}[0,15+0,15+0,2+0,55]=0,2625
\end{aligned}
$$


$\varphi 4^{-}=\frac{1}{5-1}[0,6+0,12+0,7+0,6]=0,505$

Finally calculating the Net Flow, using the 11th equation, is shown in table 6.

Table 6. Net Flow

\begin{tabular}{cccc}
\hline Alternative & $\begin{array}{c}\text { Leaving } \\
\text { Flow }\end{array}$ & $\begin{array}{c}\text { Entering } \\
\text { Flow }\end{array}$ & $\begin{array}{c}\text { Net } \\
\text { Flow }\end{array}$ \\
\hline $\mathrm{A}_{1}$ & 0,4325 & 0,4625 & $-0,030$ \\
$\mathrm{~A}_{2}$ & 0,2675 & 0,585 & $-0,318$ \\
$\mathrm{~A}_{3}$ & 0,495 & 0,65 & $-0,155$ \\
$\mathrm{~A}_{4}$ & 0,545 & 0,2625 & 0,283 \\
$\mathrm{~A}_{5}$ & 0,725 & 0,505 & 0,220 \\
\hline
\end{tabular}

The ranking or final result is shown in table 7.

Table 7. Rangking

\begin{tabular}{ccc}
\hline Alternative & Result & Rank \\
\hline $\mathrm{A}_{4}$ & 0,283 & 1 \\
$\mathrm{~A}_{5}$ & 0,220 & 2 \\
$\mathrm{~A}_{1}$ & $-0,030$ & 3 \\
$\mathrm{~A}_{3}$ & $-0,155$ & 4 \\
$\mathrm{~A}_{2}$ & $-0,318$ & 5 \\
\hline
\end{tabular}

The calculation steps apply the EXPROM II method, resulting in Muhammad Rifqy $\left(\mathrm{A}_{4}\right)$ being a student selected to participate in a student learner program at the international level with a score of 0.283 , the highest of the other four (4) alternatives.

\section{Conclusion}

The results concluded that:

1. Application of cost and benefit criterion type only occurs when normalizing xij matrix, into the rij matrix.

2. EXPROM II calculation steps more accurate and effective, because of the comparison between each pair of criteria, so the result is better, but this has a lot of comparison process.

3. EXPROM II becomes the right calculation solution for the decision maker.

\section{Reference}

[1] P. Poningsih, "Design of the expert system to analyze disease in Plant Teak using Forward Chaining," Int. J. Artif. Intell. Res., vol. 1, no. 1, p. 11, Jun. 2017.

[2] P. harliana and R. Rahim, "Comparative Analysis of Membership Function on Mamdani Fuzzy Inference System for Decision Making,” J. Phys. Conf. Ser., vol. 930, no. 1, p. 012029, Dec. 2017.

[3] N. Nurmalini and R. Rahim, "Study Approach of Simple Additive Weighting For Decision Support System,” Int. J. Sci. Res. Sci. Technol., vol. 3, no. 3, pp. 541-544, 2017.

[4] J. Jasri, D. Siregar, and R. Rahim, "Decision Support System Best Employee Assessments with 
Technique for Order of Preference by Similarity to Ideal Solution," Int. J. Recent Trends Eng. Res., vol. 3, no. 3, pp. 6-17, Mar. 2017.

[5] S. Syamsudin and R. Rahim, "Study Approach Technique for Order of Preference by Similarity to Ideal Solution (TOPSIS)," Int. J. Recent Trends Eng. Res., vol. 3, no. 3, pp. 268-285, Apr. 2017.

[6] G. Ginting, Fadlina, Mesran, A. P. U. Siahaan, and R. Rahim, "Technical Approach of TOPSIS in Decision Making," Int. J. Recent Trends Eng. Res., vol. 3, no. 8, pp. 58-64, 2017.

[7] R. Rahim, Mesran, A. Putera, U. Siahaan, and S. Aryza, "Composite performance index for student admission," Int. J. Res. Sci. Eng., vol. 3, no. 3, pp. 68-74, 2017.

[8] D. Siregar, D. Arisandi, A. Usman, D. Irwan, and R. Rahim, "Research of Simple MultiAttribute Rating Technique for Decision Support," J. Phys. Conf. Ser., vol. 930, no. 1, p. 012015, Dec. 2017.

[9] Fadlina, L. T. Sianturi, A. Karim, Mesran, and A. P. U. Siahaan, "Best Student Selection Using Extended Promethee II Method," Int. J. Recent Trends Eng. Res., vol. 3, no. 8, pp. 21-29, 2017.

[10] M. Behzadian, R. B. Kazemzadeh, A. Albadvi, and M. Aghdasi, "PROMETHEE: A comprehensive literature review on methodologies and applications," Eur. J. Oper. Res., vol. 200, no. 1, pp. 198-215, 2010.

[11] R. Attri and S. Grover, "Application of preference selection index method for decision making over the design stage of production system life cycle," J. King Saud Univ. - Eng. Sci., vol. 27, no. 2, pp. 207-216, 2015.

[12] M. Madić, J. Antucheviciene, M. Radovanović, and D. Petković, "Determination of laser cutting process conditions using the preference selection index method," Opt. Laser Technol., vol. 89, no. October 2016, pp. 214-220, 2017.

[13] T. Mufizar, T. Nuraen, and A. Salama, "Sistem Pendukung Keputusan Dalam Penentuan Pertukaran Pelajar Di Sma Negeri 2 Tasikmalaya Dengan Metode Analytical Hierarchy Process ( Ahp )," Cogito Smart J., vol. 3, no. 1, pp. 68-82, 2017.

[14] Y. Silalahi, Mesran, T. Zebua, and Suginam, "PENERAPAN THE EXTENDED PROMETHEE II ( EXPROM II ) UNTUK PENENTUAN PRODUK DISKON,” KOMIK (Konferensi Nas. Teknol. Inf. dan Komputer), vol. I, no. 1, 2017.

[15] R. Rahim, S. Nurarif, M. Ramadhan, S. Aisyah, and W. Purba, "Comparison Searching Process of Linear, Binary and Interpolation Algorithm,” J. Phys. Conf. Ser., vol. 930, no. 1, p. 012007, Dec. 2017.

[16] R. Rahim, I. Zulkarnain, and H. Jaya, "A review: search visualization with Knuth Morris Pratt algorithm,” IOP Conf. Ser. Mater. Sci. Eng., vol. 237, p. 012026, Sep. 2017.

[17] R. Rahim, I. Zulkarnain, and H. Jaya, "Double hashing technique in closed hashing search process," IOP Conf. Ser. Mater. Sci. Eng., vol. 237, p. 012027, Sep. 2017.

[18] P. Chatterjee and S. Chakraborty, "Flexible manufacturing system selection using preference ranking methods: A comparative study," Int. J. Ind. Eng. Comput., vol. 5, no. 2, pp. 315-338, 2014. 